\title{
Throughput Modeling of Single Hop CSMA Networks with Non-Negligible Propagation Delay
}

\author{
Mehmet Koseoglu and Ezhan Karasan, Member, IEEE
}

\begin{abstract}
We analyze the performance of the CSMA protocol under propagation delays that are comparable with packet transmission times. We propose a semi-Markov model for the 2-node CSMA channel. For the 2-node case, the capacity reduces to $40 \%$ of the zero-delay capacity when the one-way propagation delay is $10 \%$ of the packet transmission time. We then extend this model and obtain the optimum symmetric probing rate that achieves the maximum network throughput as a function of the average propagation delay, $\bar{d}$, and the number of nodes sharing the channel, $N$. The proposed model predicts that the total capacity decreases with $\bar{d}^{-1}$ as $N$ goes to infinity when all nodes probe the channel at the optimum rate. The optimum probing rate for each node decreases with $1 / N$ and the total optimum probing rate decreases faster than $\bar{d}^{-1}$ as $N$ goes to infinity. We investigate how the short-term unfairness problem in CSMA worsens as the propagation delay increases and propose a back-off mechanism to mitigate this issue. The theoretical results presented in this paper can be used as a benchmark for the performance improvements provided by algorithms that have already been developed.
\end{abstract}

Index Terms-Wireless networks; multiaccess communication; Carrier Sense Multiple Access (CSMA); large propagation delay.

\section{INTRODUCTION}

C ARRIER Sense Multiple Access (CSMA) is a distributed multiple access protocol which does not require cooperation between nodes. Due to its simple nature, CSMA forms the basis of many communication standards. One of the main drawbacks of the CSMA protocol is the collisions which may occur as a result of the propagation delay between nodes. In the current wireless configurations, however, propagation delay is not considered as a significant problem because it is negligible in comparison to the transmission times. On the other hand, larger propagation delays should be considered in the performance modeling of future wireless networks for several reasons: First, there are new wireless networks developed for covering larger areas to provide Internet access in rural areas [1] where the propagation delay is larger. Second, there is an emerging need for underwater acoustic networks [2] which experience very large propagation delays due to low propagation speed of acoustic waves. Finally, as the transmission rates increase, packet durations decrease, consequently, ratio of the propagation delay to the transmission time increases.

Manuscript received January 2, 2013; revised March 13, 2013. The editor coordinating the review of this paper and approving it for publication was D. I. Kim.

M. Koseoglu an E. Karasan are with the Department of Electrical and Electronics Engineering, Bilkent University, TR-06800 Bilkent, Ankara, Turkey (e-mail: kmehmet@ee.bilkent.edu.tr, ezhan@ee.bilkent.edu.tr).

Digital Object Identifier 10.1109/TCOMM.2013.050813.130004
The IEEE 802.22 wireless regional area network (WRAN) standard is developed for providing Internet access to rural areas where deploying a cable infrastructure is expensive. In this standard, a base station has a coverage range up to 100 $\mathrm{km}$ in radius [1]. The propagation delay of radio waves over a distance of $100 \mathrm{~km}$ equals to $334 \mu \mathrm{s}$ which is larger than the transmission delay of a 2000 byte packet at rates exceeding 48 Mbps. Although the IEEE 802.22 standard specifies a centralized access mechanism, propagation delay has to be taken into account if new regional wireless networks using a random access based multiple access control (MAC) scheme are to be deployed in the future. The effect of the propagation delay on the underwater acoustic networks is even more dramatic: The propagation delay of a packet over a distance of $1 \mathrm{~km}$ is $670 \mathrm{~ms}$ which is larger than the transmission delay of a 2000 byte packet at rates exceeding $24 \mathrm{Kbps}$. So, propagation delay must be a major consideration for terrestrial wireless networks covering large distances and for underwater networks even for short distances and low transmission rates.

We here model the throughputs of nodes sharing a single CSMA channel under non-negligible propagation delays. We determine how aggressive nodes should be in order to optimize the trade-off between the channel utilization and the collision probability. We also investigate the asymptotic behavior of the capacity region as the propagation delay and the number of nodes increase. Although the effects of the propagation delay have been studied in the context of practical configurations [3], [4], [5], fundamental capacity limits of the pure CSMA protocol have not been previously considered to the best of our knowledge. The contributions of the paper are:

- a semi-Markov model for the throughput of the two saturated nodes sharing a CSMA channel. Using this model, we present the capacity region of the CSMA channel with non-zero propagation delay. When the propagation delay is $10 \%$ of the packet transmission time, the capacity reduces to $40 \%$ of the zero-delay capacity for the 2 -node case.

- derivation of the optimum probing rates as a function of the average propagation delay, $\bar{d}$, and the number of nodes, $N$, by extending the 2-node model. The optimum probing rate maximizes the channel utilization by exploiting the balance between the collision probability and the channel utilization.

- an investigation of the asymptotic total capacity for large $N$. In the limit as $N \rightarrow \infty$, the model predicts that the total capacity changes in proportional to $\bar{d}^{-1}$. The optimum node probing rate decreases with $1 / N$ as $N \rightarrow \infty$. Moreover, the total optimum network probing rate achieved by all nodes decreases faster than $\bar{d}^{-1}$ for 
large $N$ according to the proposed model.

- an investigation of a back-off mechanism which is employed in order to mitigate the the short-term unfairness problem in CSMA. When the propagation delay increases, the capture effect in CSMA becomes more significant especially when a small number of nodes are sharing the channel. Using a back-off after each transmission, this unfairness becomes much less significant without having a throughput penalty.

- a comparison of the performance of the 802.11 channel access scheme with the proposed capacity and optimum probing rate analysis. For a simple two-node scenario, the 802.11 channel access scheme behaves closely similar with the proposed analysis for the pure CSMA as the propagation delay increases despite the discrepancies between the studied CSMA model and the 802.11 MAC protocol.

In the next section, we review the relevant literature. In Section III, we describe the scenario on which we built our study. The semi-Markov model for the 2-node case and the capacity region of the CSMA link are presented in Section IV. Derivation of the asymptotic optimum probing rate and the total channel capacity along with the performance evaluation of these expressions are discussed in Section V. We investigate a back-off mechanism for imroving the fairness of CSMA under large propagation delay in Section VI. Section VII compares the 802.11 channel access scheme with the proposed capacity and optimum probing rate analysis.

\section{RELATED WORK}

\section{A. Throughput-optimal Multiple Access}

Recently, it has been shown that throughput optimality in a CSMA based wireless network can be achieved by using an adaptive and distributed probing rate adaptation algorithm [6]. Because of the zero-collision assumption in this algorithm, channel access rates of nodes are allowed to approach infinity in order to achieve the limits of the throughput region. However, increasing rates indefinitely will cause zero throughput in practice because of collisions. Several studies explain how throughput optimality can be attained in case of collisions [7], [8], but the collision probability must approach to zero to achieve throughput optimality. This condition requires nodes not to be aggressive to capture the channel resulting in large channel holding times. Hence, it is not possible to achieve throughput optimality without sacrificing the shorttime fairness [9]. We here investigate how much of the zerocollision capacity can be achieved without increasing channel holding times under non-negligible propagation delays.

\section{B. Performance of Random Access under Large Propagation Delay}

Performance analysis of unslotted CSMA was given by Kleinrock and Tobagi [10]. Since this analysis is based on the infinite number of users assumption [11], the throughput expression does not provide accurate results for small number of users. For finite number of users, Takagi and Kleinrock analyzed persistent CSMA [12]. This analysis is valid only for persistent CSMA and relies on the assumption that each user has independent and exponentially distributed idle periods.

The effects of the propagation delay on the performance of CSMA in terrestrial wireless networks have not been extensively studied so far because of the short communication ranges of wireless communication technologies where CSMA is employed. Current wireless standards are based on the assumption that packet transmission times are much larger than the propagation delay. The performance of the 802.11 protocol for outdoor networks is analyzed in [13], [5] and it is stated that the 802.11 protocol is not feasible for distances larger than $6 \mathrm{~km}$. Recently, however, the 802.22 WRAN standard is proposed which has a coverage area of up to $100 \mathrm{~km}$. If random access is to be used for next generation wireless networks with larger coverage ranges, the influence of the propagation delay on the network throughput must be investigated.

The effect of the propagation delay is even more critical for underwater networks because of the relatively slow propagation speed of acoustic waves [14]. The performance of the CSMA protocol with the Request to Send / Clear to Send (RTS/CTS) mechanism under large propagation delays is investigated in [15], [16]. These studies demonstrate that the use of RTS/CTS does not improve the performance of CSMA under large propagation delays. The performance of the ALOHA protocol for underwater sensor networks working with large propagation delays is analyzed in [17], [18]. Both studies state that the performance of slotted ALOHA reduces to the performance of unslotted ALOHA under large propagation delays. Adapting slot lengths according to the propagation delay is proposed [19], [20], but using larger slot lengths reduces efficiency when the propagation delay is comparable with packet transmission times. Addition of a guard band to transmissions is proposed in [18] and an additional synchronization mechanism for slotted ALOHA is suggested in [21]. A handshaking mechanism similar to RTS/CTS is proposed for underwater networks in [3] and a cross layer approach is proposed in [22].

We investigate the performance of CSMA instead of ALOHA because channel sensing can prevent some of the collisions and can be implemented in a simple manner. We model the capacity of asynchronous CSMA without the RTS/CTS mechanism since earlier work demonstrated the ineffectiveness of the RTS/CTS mechanism and simple time synchronization under large propagation delay [16]. Although prior studies analyzed only the total channel throughput, we also present expressions for individual throughputs of nodes for the 2node case. We study how the propagation delay affects the channel capacity and what must be the optimum probing rates to achieve the capacity.

Although the collision detection mechanism used in the Ethernet protocol can potentially improve the performance of CSMA under large propagation delays since the transmitter can detect a collision much earlier, this mechanism is not feasible for wireless networks [23]. However, there are a few attempts to incorporate the collision detection mechanism for wireless networks: [24] proposed a method in which the users stop for a small amount of time after the beginning of the transmission to detect other transmissions in the channel. This 
method, which is analyzed in [25], does not allow instant collision detection because all nodes need to wait at least for the largest propagation delay in the network. For large propagation delays, this method is inefficient and it increases the complexity of the radio. A collision detection method which uses narrow-band pulses transmitted over a separate out-of-band control channel to detect collisions is proposed in [26], but addition of another channel is costly, complicating the radio hardware.

\section{SCENARIO DESCRIPTION}

In this section, we present the assumptions of this study and explain the motivations behind these assumptions.

- All nodes can hear each other, i.e., all transmissions are single hop.

- Nodes employ an unslotted CSMA protocol. We model a CSMA network where the largest one-way propagation delay can be as much as half of the packet transmission time.

- Nodes do not employ collision detection since collision detection is not feasible for wireless networks.

- Nodes do not employ any handshaking mechanism to avoid collisions. Although a handshaking mechanism may reduce packet collisions, it brings a significant overhead when the propagation delay is high. Besides, control packets used in handshaking may also collide when the propagation delay is large.

- We assume that the back-off intervals are exponentially distributed. Since the exponential distribution supports infinite back-off intervals, it is not used in real-life protocols. However, it is more suitable for the performance analysis because of its memoryless behavior. Similarly, geometric distribution is used in IEEE 802.11 performance analysis in the literature because of its memoryless nature, and it is shown to perform similar to the uniform back-off length distribution [27].

- We assume a fixed packet transmission time. Although some studies show that the throughput can be increased by increasing packet transmission times as discussed in Section II, we do not follow that approach in order to avoid degradation in the short-term fairness.

\section{Semi-Markov Model for the 2-Node CSMA CHANNEL}

In this section, we first present a throughput model for the CSMA channel for 2 nodes. Then, we compare the performance of this model with the simulation results and present the capacity region of the 2-node CSMA channel to demonstrate the effect of propagation delay on the capacity of the CSMA protocol.

\section{A. State Definitions}

The semi-Markov model for the 2-node case is built from the point of the view of one of the nodes where states represent the phases that a node visits as time evolves. The state diagram of the chain is depicted in Fig. 1.

Assume that node 1 started a transmission after a long idle period and node 1 is sharing the channel with node 2 . This

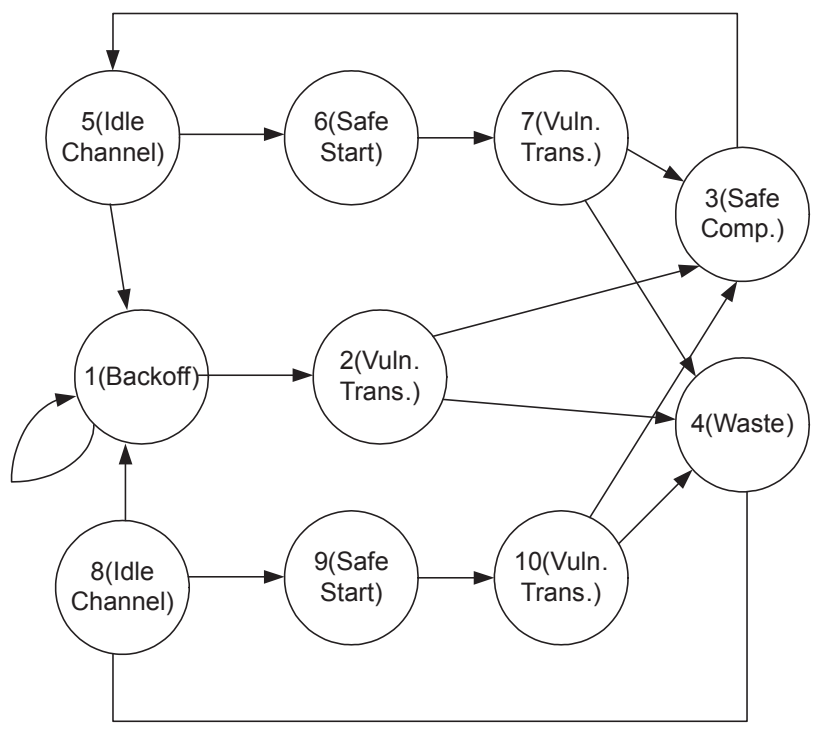

Fig. 1: The state diagram for the semi-Markov model.

transmission is vulnerable to collisions until node 2 hears this transmission (State 2). If the transmission survives this period, it is certain that the transmission will safely complete (State 3). After the end of this transmission, node 2 will still be exposed to the transmission of node 1 for a period time because of the propagation delay. During this period, node 1 is advantageous to start another transmission (State 5). If node 1 starts a transmission while node 2 is still exposed to its previous transmission, new transmission can be safe from collisions for a period of time (State 6). When this period ends, it becomes vulnerable again (State 7) but it can safely complete after node 2 hears this transmission (State 3). If a collision occurs, transmission is wasted (State 4). At the end of this collided transmission, node 2 will still be exposed to the collided transmission so a shorter period of successful probing exists (State 8). New transmission will pass through safe (State 9) and vulnerable states (State 10).

Below we present the holding time distributions and the transition probabilities between these states. We normalize the time such that each packet has a fixed transmission time of unit duration. Note that $d$ denotes the one-way propagation delay between nodes and we assume $2 d<1$. Nodes 1 and 2 independently sense the channel at exponentially distributed intervals with mean $1 / R_{1}$ and $1 / R_{2}$, respectively, and transmit their packets if the channel is idle. $R_{1}$ and $R_{2}$ refer to the probing rates of Nodes 1 and 2, respectively. $S_{i}$ denotes the holding time in state $i$. Probability distribution function (PDF) and cumulative distribution function (CDF) of $S_{i}$ are denoted by $f_{S_{i}}$ and $F_{S_{i}}$, respectively. We define $p_{i, j}$ as the transition probability from state $i$ to state $j$.

State 3 (Safe Completion): After a transmission starts, a colliding transmission can only arrive within $2 d$ period because node 2 becomes aware of the transmission of node 1 at $d$. Since node 2 will not start a transmission after this point, it is certain that a colliding transmission will not arrive after $2 d$ and the transmission will be safely completed. The 


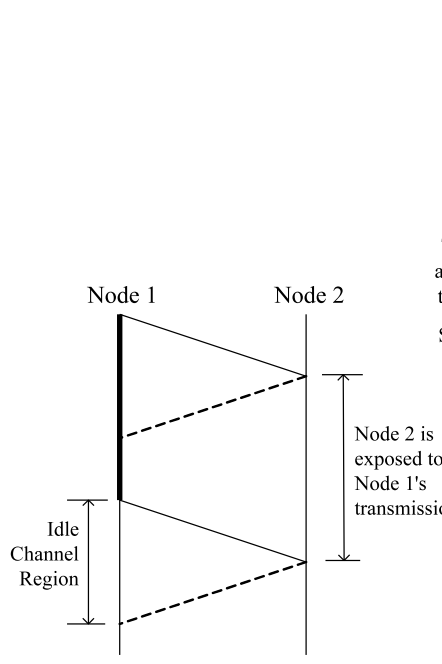

(a)

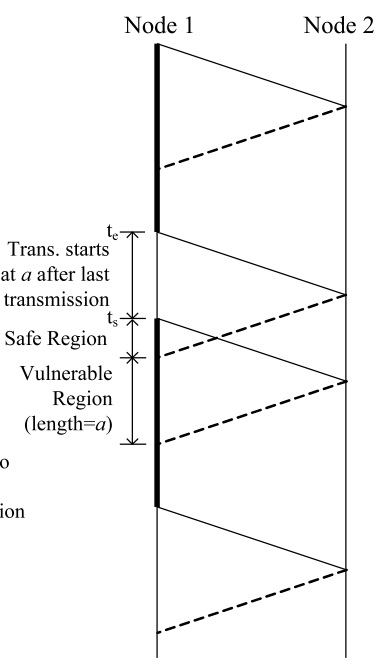

(b)
Fig. 2: (a) Idle channel period after a successful transmission. Duration of this period is $2 d$. (b) If a transmission starts in this idle period, it continues free from collisions for a duration of $a$ and enters into a vulnerable period. The duration $a$ equals to the starting transmission time after the successful transmission.

holding time in this state is deterministic and equal to $1-2 d$ :

$$
f_{S_{3}}(t)= \begin{cases}1 & \text { if } t=1-2 d, \\ 0 & \text { o.w. }\end{cases}
$$

After a successful transmission, there is an idle channel period which is the next state described. In our model, that period is denoted as State 5 and the transition probability from State 3 to State 5 is 1 , i.e.,

$$
p_{3,5}=1 \text {. }
$$

State 5 (Idle Channel): After a successful transmission, it is certain that node 1 will not receive a transmission from node 2 for a duration of $2 d$ because node 2 is still exposed to node 1's successful transmission as shown in Fig. 2a. After the successful completion, if node 1 performs channel probing within the $2 d$ duration, it will find the channel idle and start transmission and enter State 6 (Safe Start). If it does not probe the channel within the $2 d$ period, the system will enter State 1 (Backoff). Hence, the transition probabilities from State 5 are given by

$$
p_{5,1}=e^{-R_{1} 2 d} \quad p_{5,6}=1-e^{-R_{1} 2 d}
$$

and the holding time distribution in State 5 is given by

$$
f_{S_{5}}(t)= \begin{cases}R_{1} e^{-R_{1} t} & \text { if } t<2 d, \\ e^{-R_{1} 2 d} & \text { if } t=2 d, \\ 0 & \text { o.w. }\end{cases}
$$

Then, the expected holding time at State 5 can be written as

$$
E\left[S_{5}\right]=\int_{0}^{2 d} t R_{1} e^{-R_{1} t} d t+2 d e^{-R_{1} 2 d}=\frac{1-e^{-R_{1} 2 d}}{R_{1}} .
$$

State 6 (Safe Start): If node 1 starts transmission within the $2 d$ period, it is certain that this transmission will safely continue until time $t_{e}+2 d$, where $t_{e}$ is the end of last packet transmission as it can be observed from Fig. 2b. After this

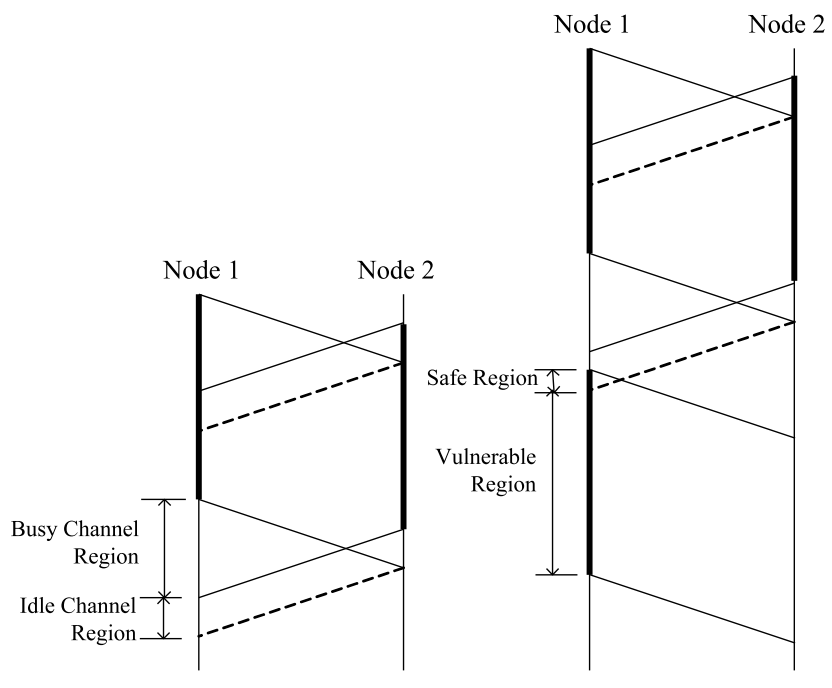

(a)

(b)

Fig. 3: (a) Busy and idle channel periods after an unsuccessful transmission. (b) If a transmission starts in the idle period, it continues free from collisions for a while and enters into a vulnerable period.

state, the transmission will enter a vulnerable state (State 7):

$$
p_{6,7}=1 \text {. }
$$

The holding time distribution in this state is given by

$$
f_{S_{6}}(t)= \begin{cases}\frac{R_{1} e^{-R_{1}(2 d-t)}}{1-e^{-R_{1} 2 d}} & \text { if } t<2 d \\ 0 & \text { o.w. }\end{cases}
$$

Then,

$$
E\left[S_{6}\right]=\int_{0}^{2 d} t \frac{R_{1} e^{-R_{1}(2 d-t)}}{1-e^{-R_{1} 2 d}} d t=-\frac{1}{R_{1}}+d+d \operatorname{coth}\left(R_{1} d\right) .
$$

State 7 (Vulnerable Transmission): After State 6, the transmission becomes vulnerable in $\left[t_{e}+2 d, t_{s}+2 d\right]$ as shown in Fig. $2 \mathrm{~b}$, where $t_{s}$ is the starting time of the transmission of the current packet. As noted in the figure, the length of the vulnerable period is equal to the starting time of the transmission after the last transmission. For that reason, the length of this period is exponentially distributed truncated at $2 d$. Then, the probability of successful completion of the transmission, which corresponds to the probability of transition to State 3, is expressed as

$$
\begin{aligned}
p_{7,3} & =\int_{0}^{2 d} \frac{R_{1} e^{-R_{1} t}}{1-e^{-R_{1} 2 d}} e^{-R_{2} t} d t \\
& =\frac{e^{-R_{2} d} R_{1} \operatorname{csch}\left(R_{1} d\right) \sinh \left(\left(R_{1}+R_{2}\right) d\right)}{R_{1}+R_{2}} .
\end{aligned}
$$

Consequently,

$$
p_{7,4}=1-p_{7,3} .
$$

In order to obtain the holding time distribution of this state, the distribution of the minimum of two random variables has to be found. Either the vulnerable period will end without collisions and the system will enter State 3 or a colliding transmission will arrive and the system will enter State 4 . The first distribution which denotes the length of the vulnerable period, $V$, is exponentially distributed truncated at $2 d$. The 
second distribution is the distribution of the arrival of the other node's transmission, $C$, which is exponentially distributed with mean $1 / R_{2}$.

$$
F_{S_{7}}(t)= \begin{cases}1-\left(\int_{t}^{2 d} \frac{R_{1} e^{-R_{1} x}}{1-e^{-R_{1} 2 d}} d x\right) e^{-R_{2} t} & t<2 d \\ 1 & \text { o.w. }\end{cases}
$$

Taking the derivative, $f_{S_{7}}(t)$ can be obtained:

$$
f_{S_{7}}(t)= \begin{cases}-\frac{e^{-a R_{1}-a R_{2}+2 R_{1} d} R_{1}}{1-e^{2 R_{1} d}}+\frac{e^{-a R_{2}}\left(1-e^{-a R_{1}+2 R_{1} d}\right) R_{2}}{1-e^{2 R_{1} d}} & t<2 d \\ 0 & \text { o.w. }\end{cases}
$$

The expected length of this period is given by

$$
E\left[S_{7}\right]=\int_{0}^{2 d} t f_{S_{7}}(t) d t=\frac{\left(-1+e^{-2 R_{2} d}\right) R_{1}+\left(-1+e^{2 R_{1} d}\right) R_{2}}{\left(-1+e^{2 R_{1} d}\right) R_{2}\left(R_{1}+R_{2}\right)} .
$$

State 4 (Waste): If a colliding transmission arrives during the vulnerable period of a transmission, the system will enter State 4 . The duration of this period equals 1 which is the length of the colliding transmission, hence $E\left[S_{4}\right]=1$. After State 4 , the system will enter an idle waiting state (State 8): $p_{4,8}=1$.

State 8 (Idle Channel): After State 4, there is still an idle period during which a probe will be successful as it can be observed in Fig. 3a. However, this period will be shorter than $2 d$ in contrast to State 5. The length of this period is given by $2 d-t_{c}$ where $t_{c}$ is the duration of the collision after the previous transmission. We assume that the collision duration is uniformly distributed in $[0,2 d]$. Then, the probability that the node probes the channel before the end of the idle period, i.e., the transition probability from State 8 to State 9 , can be expressed as

$$
p_{8,9}=\int_{0}^{2 d} \frac{1}{2 d} \int_{0}^{u} R_{1} e^{-x R_{1}} d x d u=1-\frac{1-e^{-R_{1} 2 d}}{2 R_{1} d}
$$

and $p_{8,1}=1-p_{8,9}$. The distribution of the holding time in State 8 is the minimum of two random variables. The first one is the length of the idle period which is uniformly distributed between 0 and $2 d$. The other one is the probing time which is exponentially distributed with mean $1 / R_{1}$.

$$
F_{S_{8}}(t)= \begin{cases}\left(1-e^{-R_{1} t} \frac{2 d-t}{2 d}\right) & t<2 d \\ 1 & \text { o.w. }\end{cases}
$$

Taking the derivative, $f_{S_{8}}(t)$ can be written as

$$
f_{S_{8}}(t)= \begin{cases}\frac{e^{-R_{1} t}}{2 d}+\frac{e^{-R_{1} t} R_{1}(-t+2 d)}{2 d} & t<2 d \\ 0 & \text { o.w. }\end{cases}
$$

The expected holding time at State 8 is given by

$$
E\left[S_{8}\right]=\int_{0}^{2 d} t f_{S_{8}}(t) d t=-\frac{1-e^{-2 R_{1} d}-2 R_{1} d}{2 R_{1}^{2} d} .
$$

State 9 (Safe Start): If the node probes the channel in the idle period after an unsuccessful transmission, the started transmission will continue safely for a while as shown in Fig. 3b. Let $U$ denote the length of the idle period which is uniformly distributed between 0 and $2 d$ and $E$ is the starting time of the transmission which is exponentially distributed with mean $1 / R_{1}$. Since the length of the idle period is $U-E$, the $\mathrm{CDF}$ of $S_{9}$ can be written as

$$
\begin{aligned}
F_{S_{9}}(t)= & \operatorname{Pr}(U-E<t \mid E<U) \\
= & \operatorname{Pr}(U-E<t \mid E<U, U<t)+ \\
& \quad \operatorname{Pr}(U-E<t \mid E<U, U>t) \\
= & \operatorname{Pr}(U<t)+\operatorname{Pr}(U-E<t \mid E<U, U>t) \\
= & \frac{t}{2 d}+\int_{0}^{2 d} \frac{1}{2 d} \int_{u-t}^{u} R_{1} e^{-R_{1} t} d t d u \\
= & \frac{t+\frac{1-e^{-R_{1} t}-e^{R_{1}(t-2 d)}+e^{-R_{1} 2 d}}{R_{1}}}{2 d}
\end{aligned}
$$

Then, $f_{S_{9}}(t)$ is given by

$$
f_{S_{9}}(t)=\frac{1+e^{-R_{1} t}-e^{R_{1}(t-2 d)}}{2 d}
$$

and the expected holding time is expressed as

$$
\begin{aligned}
E\left[S_{9}\right] & =\int_{0}^{2 d} t f_{S_{9}}(t) d t \\
& =\frac{1+R_{1} d\left(-1+R_{1} d\right)-e^{-R_{1} 2 d}\left(1+R_{1} d\right)}{R_{1}^{2} d} .
\end{aligned}
$$

After visiting State 9, the system will enter State 10: $p_{9,10}=1$.

State 10 (Vulnerable Period): After State 9, there is a vulnerable period during which a collision may occur as it can be observed from Fig. 3b. Distribution of the holding time of State 10 is the minimum of two distributions: The first one is the maximum duration of this period which is the subtraction of the holding time in State 9 from $2 d$ and the second one is exponentially distributed with mean $1 / R_{2}$ which corresponds to the duration until the start of a colliding transmission. Probability that a colliding transmission arrives during a transmission can be written as

$$
\begin{aligned}
p_{10,4}= & \int_{0}^{2 d} \frac{1+e^{-(2 d-u) R_{1}}-e^{R_{1}(-u)}}{2 d} \int_{0}^{u} R_{2} e^{-R_{2} t} d t d u \\
= & 1+\frac{e^{-2 d R_{1}}}{2 d\left(R_{1}-R_{2}\right)}+\frac{e^{-2 d R_{2}}}{2 d R_{2}}+\frac{e^{-2 d R_{2}}}{2 d\left(-R_{1}+R_{2}\right)} \\
& \quad-\frac{e^{-2 d\left(R_{1}+R_{2}\right)}}{2 d\left(R_{1}+R_{2}\right)}-\frac{R_{1}}{2 d\left(R_{1} R_{2}+R_{2}^{2}\right)}
\end{aligned}
$$

and $p_{10,3}=1-p_{10,4}$. The holding time cumulative distribution function, $F_{S_{10}}(t)$, is given by

$$
\begin{aligned}
F_{S_{10}}(t)=1 & -e^{-R_{2} t} \int_{t}^{2 d} \frac{1+e^{-R_{1}(2 d-t)}-e^{R_{1}(-t)}}{2 d} \\
=1 & -e^{-R_{2} t}-\frac{e^{-R_{2} t}}{2 d R_{1}}-\frac{e^{-2 d R_{1}-R_{2} t}}{2 d R_{1}} \\
& \quad+\frac{e^{-R_{1} t-R_{2} t}}{2 d R_{1}}+\frac{e^{-R_{2} t+R_{1}(-2 d+t)}}{2 d R_{1}}+\frac{e^{-R_{2} t} t}{2 d}
\end{aligned}
$$

Then, the expected holding time is given by

$$
\begin{aligned}
E\left[S_{10}\right]= & \int_{0}^{2 d} t f_{S_{10}}(t) d t \\
= & \frac{1}{2 d R_{2}^{2}\left(-R_{1}^{2}+R_{2}^{2}\right)} e^{-2 d\left(R_{1}+R_{2}\right)} \\
& \left(\left(R_{1}-R_{2}\right) R_{2}-e^{2 d R_{1}}\left(R_{1}-2 R_{2}\right)\left(R_{1}+R_{2}\right)\right. \\
& \quad-e^{2 d R_{2}} R_{2}\left(R_{1}+R_{2}\right) \\
& \left.+e^{2 d\left(R_{1}+R_{2}\right)}\left(R_{1}^{2}-R_{1}\left(1+2 d R_{1}\right) R_{2}+2 d R_{2}^{3}\right)\right) .
\end{aligned}
$$


State 1 (Backoff): If the node does not probe the channel in the idle periods after successful or unsuccessful transmissions, the system will enter the backoff state. In this state, the successful probing probability (i.e. the probability of finding the channel idle at the time of probing) is reduced because the other node's transmission could have been already started before the node probes the channel. If the node finds the channel busy, the system will make a self-transition to this state. Although the probability of finding the channel idle depends on the previous state, we assume it is independent of the previous states and express the successful probing probability as

$$
p_{1,2}=\frac{\frac{1}{R_{2}}}{\frac{1}{R_{2}}+1}
$$

which is the ratio of the expected waiting time over whole time. The expected holding time in this state is

$$
E\left[S_{1}\right]=\frac{1}{R_{1}}
$$

and $p_{1,1}=1-p_{1,2}$.

State 2 (Vulnerable Transmission): If the node finds the channel idle at State 1, it starts a transmission. This transmission will be vulnerable to other node's transmission from the beginning since it does not start immediately after a transmission. So, probability of transition from State 2 to State 3 and 4 can be written as

$$
p_{2,3}=e^{-R_{2} 2 d} \quad p_{2,4}=1-e^{-R_{2} 2 d} .
$$

Then, the holding time distribution in State 2 is given by

$$
f_{S_{2}}(t)= \begin{cases}R_{2} e^{-R_{2} t} & \text { if } t<2 d, \\ e^{-R_{2} 2 d} & \text { if } t=2 d, \\ 0 & \text { o.w. }\end{cases}
$$

Then, the expected holding time at State 2 can be written as

$$
E\left[S_{2}\right]=\int_{0}^{2 d} t R_{2} e^{-R_{2} t} d t+2 d e^{-R_{2} 2 d}=\frac{1-e^{-R_{2} 2 d}}{R_{2}} .
$$

Throughput Expression: The transition matrix of the jump-chain of the semi-Markov model shown in Fig. 1 is given by

$$
P=\left(\begin{array}{cccccccccc}
p_{1,1} & p_{1,2} & 0 & 0 & 0 & 0 & 0 & 0 & 0 & 0 \\
0 & 0 & p_{2,3} & p_{2,4} & 0 & 0 & 0 & 0 & 0 & 0 \\
0 & 0 & 0 & 0 & 1 & 0 & 0 & 0 & 0 & 0 \\
0 & 0 & 0 & 0 & 0 & 0 & 0 & 1 & 0 & 0 \\
p_{5,1} & 0 & 0 & 0 & 0 & p_{5,6} & 0 & 0 & 0 & 0 \\
0 & 0 & 0 & 0 & 0 & 0 & 1 & 0 & 0 & 0 \\
0 & 0 & p_{7,3} & p_{7,4} & 0 & 0 & 0 & 0 & 0 & 0 \\
p_{8,1} & 0 & 0 & 0 & 0 & 0 & 0 & 0 & p_{8,9} & 0 \\
0 & 0 & 0 & 0 & 0 & 0 & 0 & 0 & 0 & 1 \\
0 & 0 & p_{10,3} & p_{10,4} & 0 & 0 & 0 & 0 & 0 & 0
\end{array}\right) .
$$

The steady-state probability distribution of the jump chain with a transition matrix $P$ is a $1 \times 10$ vector, $\pi$, and it can be obtained by solving

$$
\begin{array}{r}
\pi=\pi P \\
\sum_{i} \pi_{i}=1 .
\end{array}
$$

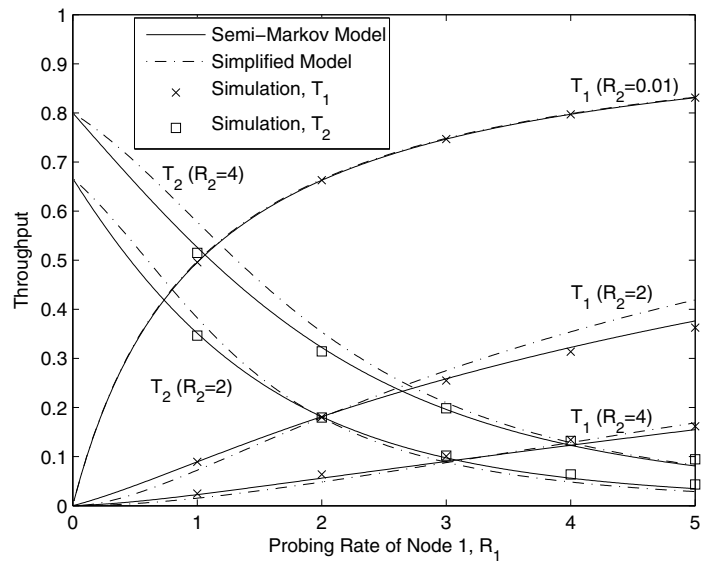

Fig. 4: Performance of the semi-Markov model and the simplified model as $R_{1}$ changes for $d=0.4$.

since the stationary probability vector, $\pi$, remains same despite the multiplication of the transition matrix. Let $T_{1}$ and $T_{2}$ denote throughputs of node 1 and node 2, respectively. Since the duration of a successful transmission is 1 and $\pi_{3}$ gives the successful transmission probability, $T_{1}$ can be written as

$$
T_{1}\left(R_{1}, R_{2}, d\right)=\frac{\pi_{3}}{\sum_{i} \pi_{i} E\left[S_{i}\right]}
$$

Although the throughput has a closed-form expression, space limitations prevent us from presenting the full expression. The model computes the throughput very accurately as it will be shown next through numerical examples.

\section{B. Accuracy of the Model}

We evaluate the performance of the semi-Markov model for the 2-node case. The simulations are performed by a selfdeveloped simulation software based on Java for a duration of $10^{6}$ time units where a transmission lasts for 1 time unit.

Fig. 4 depicts the throughputs of nodes in a 2-node network as a function of $R_{1}$ for $d=0.4$. Different plots on the graph correspond to different values of $R_{2}$. As it can be observed, the semi-Markov model accurately predicts the throughput. Maximum absolute error in throughput between the model and the simulations is 0.02 , which shows that the assumptions made in deriving the holding time distributions of State 1 and 8 have minor effects on the accuracy of the model.

\section{The Capacity Region of the CSMA Channel for $N=2$}

In this part, we provide the capacity region of the CSMA channel under non-zero propagation delay. Fig. 5a shows the maximum achievable throughputs of the two nodes sharing a CSMA channel as $d$ increases. This graph is obtained by numerical maximization of the throughput function obtained by the semi-Markov model.

It is theoretically possible to achieve the full capacity region for the zero-delay channel by probing the channel at an infinite rate. In the zero-delay case, all throughput pairs $T_{1}+T_{2} \leq$ 1 can be achieved. However, the capacity region shrinks as $d$ increases. This reduction is more apparent if nodes probe the channel at similar rates as the wasted capacity increases 


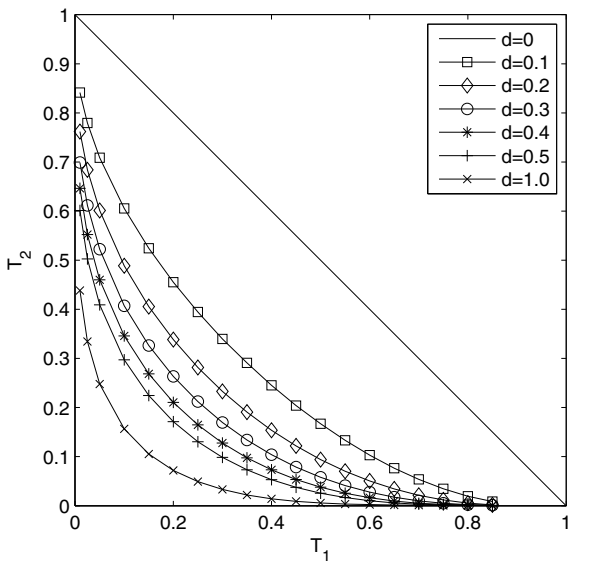

(a)

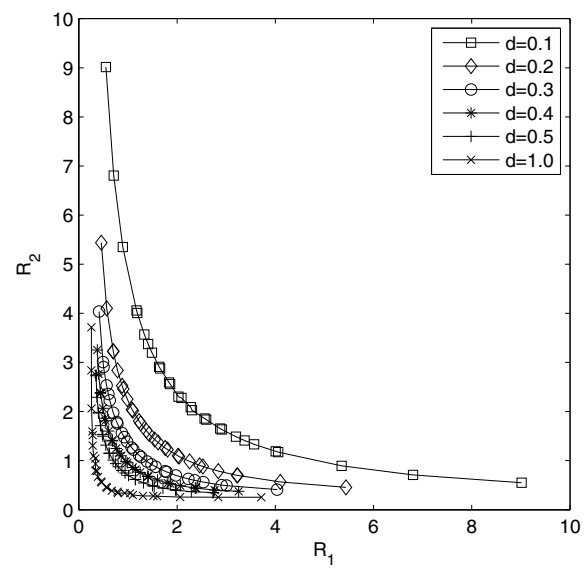

(b)

Fig. 5: (a) The capacity region of a CSMA channel with two-nodes for different propagation delays. (b) Probing rates of nodes required to achieve the limits of the capacity region.

due to collisions. On the contrary, total achievable throughput increases if one of the nodes dominates the channel because the dominant node experiences fewer collisions.

Fig. $5 \mathrm{~b}$ shows the optimum probing rates of nodes that achieve the maximum capacity as the propagation delay changes. The graph shows that nodes should probe the channel less aggressively if the propagation delay is large because of higher collision probability. Also, it can be seen that the optimum probing rate of a node is dependent on the probing rate of the other node. Nodes should be less aggressive if both nodes try to achieve similar throughputs. On the other hand, an increase in the probing rate is beneficial only if the other node probes the channel at a low rate.

The effect of the propagation delay on the throughput can be seen in Fig. 6 for symmetric probing rate values. As the propagation delay increases, probing at a lower rate yields larger throughputs by reducing the collision probability. Probing at a higher rate, however, increases the throughput at low propagation delays by decreasing the channel access delay.

These results show the importance of network-awareness and probing rate adaptation when the propagation delay is non-negligible. If several nodes sharing a channel have high throughput demands, they must be cautious not to probe the channel too frequently in order not to increase collisions. The distributed probing rate adaptation algorithm proposed in [6] allows arbitrarily large probing rates because of the zero-delay assumption but simulations show that this approach is not optimal especially when the propagation delay is large.

\section{Asymptotic Capacity and Optimum Probing Rate}

In this section, we obtain the optimum probing rate which achieves the maximum throughput for a CSMA channel with $N$ nodes. We investigate how this optimum rate and maximum throughput changes as the average propagation delay, $\bar{d}$, and the number of nodes sharing the CSMA channel, $N$, increase.

For $N>2$, modeling interactions between nodes sharing a single channel in an asynchronous fashion becomes highly complex. Each node is exposed to the transmissions of all

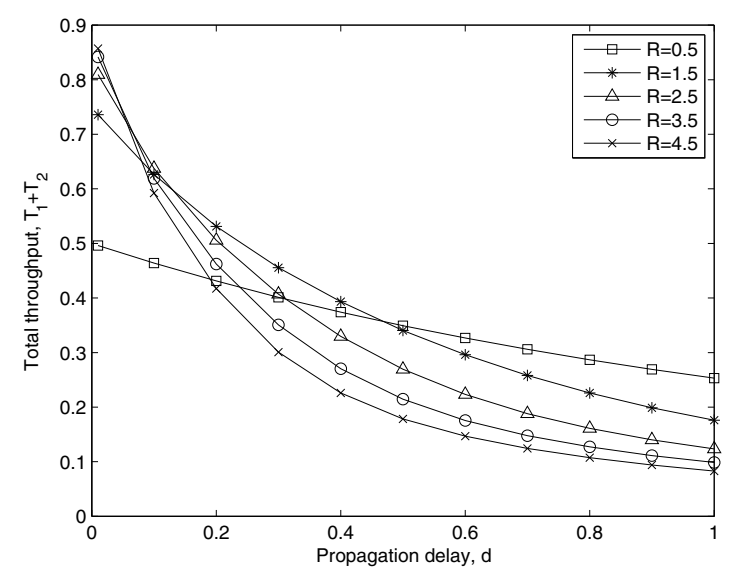

Fig. 6: Total throughput of two nodes sharing a channel as the propagation delay increases for different $R_{1}=R_{2}=R$ values.

other nodes in the channel which are also affected by the transmissions of the remaining nodes in the channel. Considering that the distances between nodes differ from each other and transmissions may start at any time, some simplifying assumptions are needed to obtain results for $N>2$. For that reason, we assume that the throughput reduction of a node caused by each neighbor is independent of other neighbors and total throughput reduction of a node can be found by multiplying individual throughput reductions stemming from each neighbor. Despite a reduction in accuracy, this approximation allows us to derive a simple expression for the channel throughput which describes how total maximum throughput and the optimum probing rate scales with $\bar{d}$ and $N$. Numerical results given at the end of this section show that the inaccuracy resulting from the above independence assumption is small and the proposed asymptotic throughput and optimum probing rate functions accurately match with the simulation results.

Next, we model the throughput reduction caused by a single neighbor of a node due to the propagation delay. 


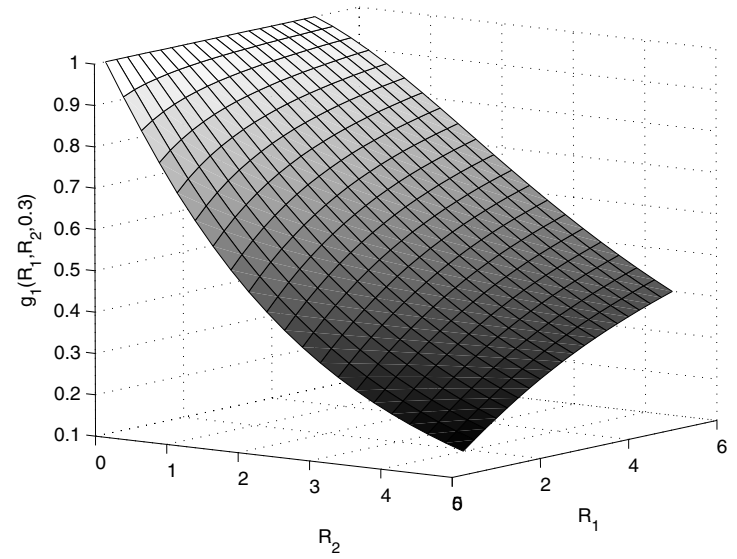

Fig. 7: $g_{1}\left(R_{1}, R_{2}, d\right)$ with changing $R_{1}$ and $R_{2}$.

\section{A. Throughput Reduction Caused by a Single Neighbor}

If the propagation delay between two nodes is 0 , throughput of node 1 is [28]

$$
T_{1}\left(R_{1}, R_{2}, 0\right)=\frac{R_{1}}{1+R_{1}+R_{2}} .
$$

To single out the effect of propagation delay, we decompose $T_{1}\left(R_{1}, R_{2}, d\right)$ into two parts:

$$
T_{1}\left(R_{1}, R_{2}, d\right)=T_{1}\left(R_{1}, R_{2}, 0\right) g_{1}\left(R_{1}, R_{2}, d\right)
$$

where $g_{1}$ represents the reduction in the throughput caused by the propagation delay due to a neighbor at distance $d$ and it can be obtained by dividing the throughput found using the semi-Markov model to the zero-delay throughput. In order to obtain a simplification for $g_{1}$, we first investigate how $g_{1}$ changes with respect to $R_{1}, R_{2}$ and $d$ using the proposed semi-Markov model. The dependence of $g_{1}$ on $d$ is intuitive: $g_{1}\left(R_{1}, R_{2}, 0\right)=1$ because there are no collisions, while $g_{1}$ decreases as $d$ increases since larger propagation delay results in higher collision probability. However, the dependences of $g_{1}$ on $R_{1}$ and $R_{2}$ are more complicated. Fig. 7 shows how $g_{1}$ changes with respect to $R_{1}$ and $R_{2}$ for $d=0.3$. The following properties can be observed from this figure:

- If $R_{2}=0, g_{1}\left(R_{1}, 0, d\right)=1$ independent of $R_{1}$. Since there are no collisions if node 2 does not probe the channel, this result is expected.

- For a given $R_{1} \neq 0, g_{1}$ decreases as $R_{2}$ increases since the ratio of collided transmissions of node 1 increases.

- For a given $R_{2} \neq 0, g_{1}$ increases as $R_{1}$ increases. Although the number of collisions that node 1 experiences increases with its probing rate, the ratio of its successful transmissions to its attempted transmissions increases, resulting in an increase in $g$.

We propose the following functional form in order to approximate $g_{1}$, which satisfies all of the above conditions

$$
\tilde{g}_{1}\left(R_{1}, R_{2}, d\right)=\frac{1}{1+k \frac{R_{2}^{b} d^{c}}{R_{1}^{a}}}
$$

where $a, b, c$ and $k$ are positive parameters representing the effect of several variables on $g_{1}$. We applied a least squares fit with integer values for $a, b$ and $c$ and obtained an approximate function which is given by

$$
\tilde{g}_{1}\left(R_{1}, R_{2}, d\right)=\frac{1}{1+k \frac{R_{2}^{2} d}{R_{1}}}
$$

where $k=1.53$. So, an approximation to $T_{1}$ is given by

$$
\tilde{T}_{1}\left(R_{1}, R_{2}, d\right)=\frac{R_{1}}{1+R_{1}+R_{2}} \frac{1}{1+k \frac{R_{2}^{2} d}{R_{1}}} .
$$

The performance of this simplified function is given in Fig. 4. Although this simplification is not as accurate as the semi-Markov model, the maximum absolute error in the throughput is limited to 0.06 . Using this model, we will now derive the asymptotic capacity and the optimum probing rate.

\section{B. Derivation of the Asymptotic Capacity and Optimum Prob- ing Rate}

Let $R$ represent the probing rate of all nodes. If there is no propagation delay in the channel, there are no collisions. Since all nodes probe the channel at exponentially distributed intervals, neighbors of a node behave as a single node. Hence, the throughput of a node is given by $T_{1}(R,(N-1) R, 0)$ where $(N-1) R$ represents the total probing rates of all other nodes. For the non-zero propagation delay case, we include the effect of each neighbor as if its effect in reducing the throughput of a node is independent from other nodes. We multiply the zero-collision throughput by the individual collision reductions $g_{1}(R, R, \bar{d})$ using the average distance for all nodes. Then, the total throughput of all nodes, $T^{A}($.$) , can be written as$

$$
T^{A}(R, \bar{d}, N)=N T_{1}(R,(N-1) R, 0)\left[g_{1}(R, R, \bar{d})\right]^{N-1} .
$$

where $g_{1}(R, R, \bar{d})$ is the throughput reduction of a node caused by another node if these two nodes were the only nodes sharing the channel. Using (35), the total throughput is approximated as

$$
T^{A}(R, \bar{d}, N) \approx \tilde{T}^{A}(R, \bar{d}, N) \triangleq N \frac{R}{1+N R}\left(\frac{1}{1+k R \bar{d}}\right)^{N-1} .
$$

The first derivative of the throughput function has a single positive root giving the optimum rate, $R^{*}$, which maximizes the throughput, $\tilde{T}^{A}$, as given by

$$
R^{*}(\bar{d}, N)=\frac{2}{k \bar{d}(N-2)+\sqrt{k \bar{d}} \sqrt{k \bar{d}(N-2)^{2}+4(N-1) N}} .
$$

Note that $R^{*}$ decreases with $1 / N$ as $N$ goes to infinity. The limit of the total optimum network probing rate achieved by all nodes as $N$ goes to infinity can be written as

$$
R^{A}(\bar{d}) \triangleq \lim _{N \rightarrow \infty} N R^{*}(\bar{d}, N)=\frac{2}{k \bar{d}+\sqrt{k \bar{d}(4+k \bar{d})}} .
$$

$R^{A}(\bar{d})$ can be bounded from below and above as given by

$$
\frac{1}{k \bar{d}+\sqrt{k \bar{d}}} \leq R^{A}(\bar{d}) \leq \frac{1}{k \bar{d}} .
$$

According to (41), the total optimum network probing rate decreases faster than $\bar{d}^{-1}$ for large $N$.

Maximum achievable throughput by a single node can be obtained by substituting (39) into (38). The limit of the total capacity achieved by all nodes as the number of nodes goes 


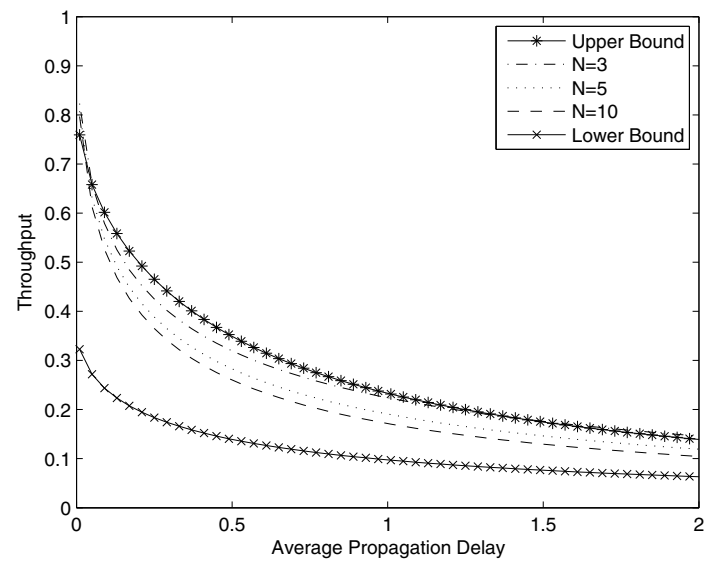

Fig. 8: Comparison of the total network throughput as a function of $\bar{d}$ for different values of $N$ along with the lower and upper bounds, $\underline{c}(\bar{d})$ and $\bar{c}(\bar{d})$.

to infinity can be written as

$$
c(\bar{d}) \triangleq \lim _{N \rightarrow \infty} \tilde{T}^{A}\left(R^{*}, \bar{d}, N\right)=\frac{2 e^{-\frac{2 k \bar{d}}{k \bar{d}+\sqrt{k \bar{d}(4+k d)}}}}{2+k \bar{d}+\sqrt{k \bar{d}(4+k \bar{d})}}
$$

and $c(\bar{d})$ can be upper and lower bounded as

$$
\underline{c}(\bar{d}) \triangleq \frac{e^{-1}}{1+k \bar{d}+\sqrt{k \bar{d}}} \leq c(\bar{d}) \leq \frac{e^{-\frac{1}{1+\frac{1}{\sqrt[4]{k d}}}}}{1+k \bar{d}} \triangleq \bar{c}(\bar{d}) .
$$

Since

$$
\lim _{\bar{d} \rightarrow \infty} \frac{\bar{c}(\bar{d})}{\underline{c}(\bar{d})}=1
$$

these bounds are asymptotically tight as $\bar{d} \rightarrow \infty$. Since the dominant term in both bounds is $\bar{d}^{-1}$, the model predicts that the total capacity decreases with $\bar{d}^{-1}$ for large $N$. Fig. 8 depicts these bounds along with the total capacity as a function of $\bar{d}$ for different number of nodes. As $N$ increases, the total capacity curve falls between the upper and lower bounds.

We now evaluate the accuracies of the total optimum probing rate and the asymptotic capacity expressions given by (40) and (42), respectively. We performed simulations for $N=10$, 25,50 and 100 by uniformly distributing nodes over a circular area whose size is determined in order to satisfy the desired average delay, $\bar{d}$. For each $N$, we conducted simulations for $\bar{d}=0.1,0.2,0.3,0.4$ and 0.5 . For each $N$ and $\bar{d}$ combination, we simulated 10 different topologies and we reported the average of the results of these simulations. For each topology, we performed 50 simulations for total probing rates between 0 and 5 with a resolution of 0.1 to obtain the optimum probing rate which maximizes the total network throughput. We denote this maximum network throughput as the network capacity. For $N=2$, we simulated a single topology with 2 nodes that are separated by $\bar{d}$ for each value of the probing rate.

The network capacity obtained by simulations for different $N$ is plotted as $\bar{d}$ increases in Fig. 9. The proposed asymptotic capacity expression given by (42) is also depicted. For large $N$, the capacity of the network approaches to the proposed asymptotic capacity. These results suggest that the capacity of

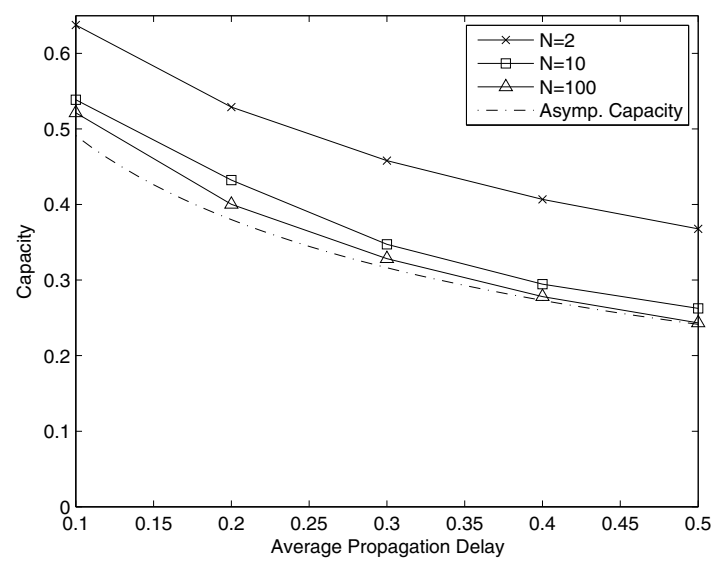

Fig. 9: The capacity of the network as $\bar{d}$ increases. The asymptotic capacity is plotted using (42).

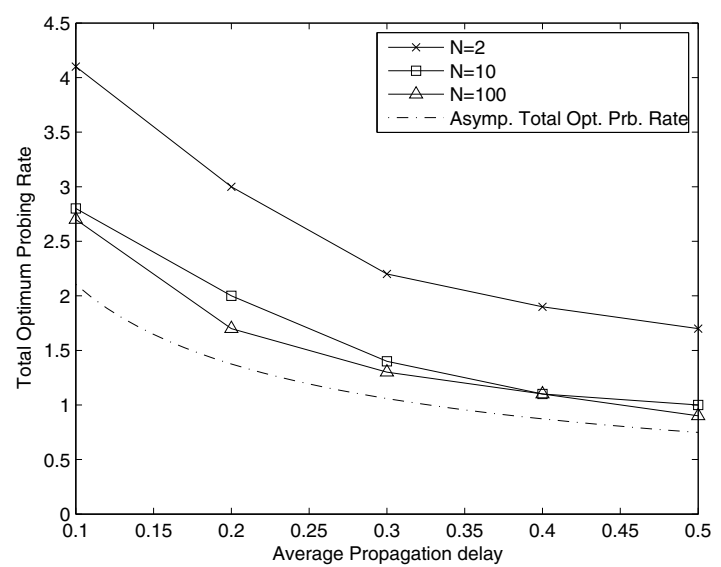

Fig. 10: Total optimum probing rate in the network as $\bar{d}$ increases. Asymptotic total optimum probing rate is plotted using (40).

the network does not degrade indefinitely as the number of nodes increases. Naturally, however, the individual throughputs of nodes degrade with $1 / N$ as nodes join the network.

Fig. 10 presents the optimum total probing rate obtained by simulations for different values of $N$ as $\bar{d}$ increases. The asymptotic optimum total probing rate given by (40) is also depicted. Our analysis indicates that the optimum total probing rate converges to an asymptotic value for large $N$ for a given $\bar{d}$. So, the nodes have to reduce their probing rates in proportion with $1 / N$ as a node enters the network to keep the total probing rate in the network constant.

These results indicate that the proposed asymptotic optimum probing rate and the capacity expressions successfully match with the simulation results for large $N$. The independence assumption made in deriving these expressions does not result in a significant inaccuracy.

\section{IMPROVING SHORT-TERM FAIRNESS IN A CSMA Channel with Non-Negligible Propagation Delay}

In a CSMA channel with non-negligible propagation delay, a node stays exposed to a completed transmission after the 


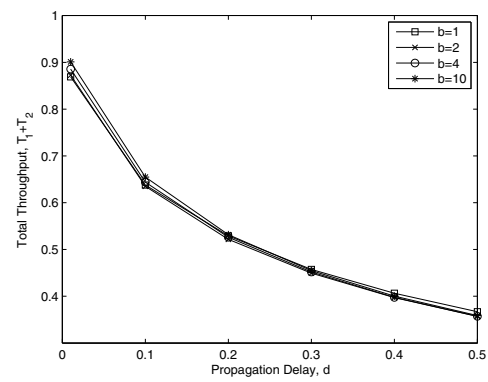

(a) $N=2$

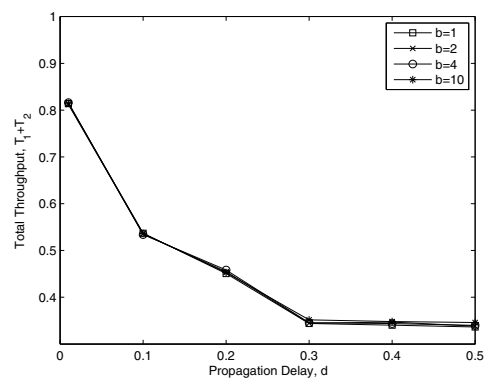

(b) $N=10$

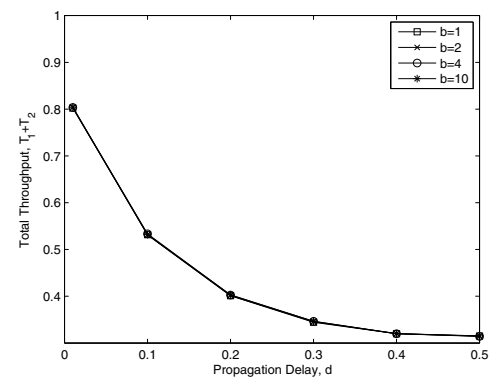

(c) $N=100$

Fig. 11: Maximum throughput achieved by the back-off scheme.

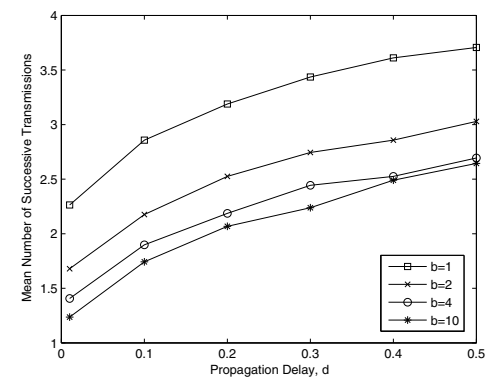

(a) $N=2$

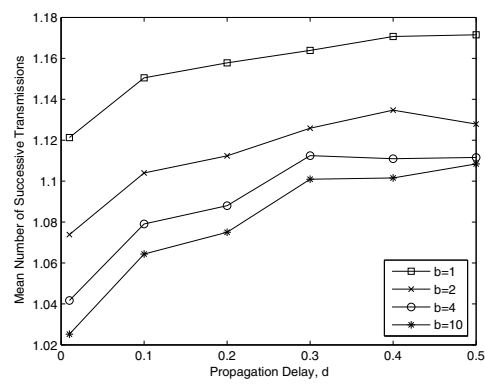

(b) $N=10$

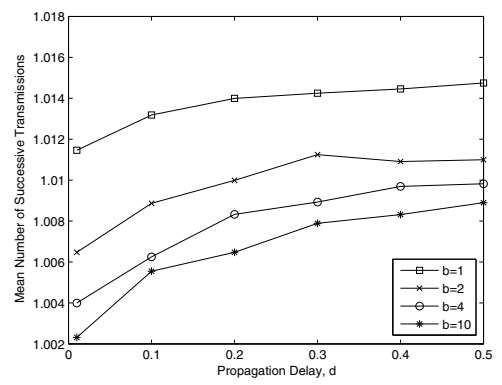

(c) $N=100$

Fig. 12: Mean number of successive transmission achieved by the back-off scheme.

transmitting node finishes the transmission. For that reason, the transmitting node finds the channel idle for some extra duration after a completed transmission so this node can start a new successive transmission if it probes the channel within this interval, i.e., while the channel is in State 5 or in State 8 in the semi-Markov model presented in Section IV. This opportunity may impair the short-term fairness of the CSMA link by allowing a node to transmit successively several times. In this section, we investigate the extent of unfairness caused by successive transmissions and propose a method to reduce the short-term unfairness.

In the proposed method, the probing rate of a transmitting node is reduced after a transmission, so that the transmitting station has a lower probability of capturing the channel. This back-off mechanism corresponds to reducing the probing rate of the node after completing a transmission when the node is in States 5 or 8 in the semi-Markov CSMA model. The reduction is performed both after a successful transmission and a collided transmission, since the node cannot immediately know whether the completed transmission is successful at the end of its transmission.

As the short-term fairness metric, we use the mean number of successive transmissions that a node makes when it captures the channel [29]. The throughput performance of the proposed method can be evaluated by making modifications on the analytical model presented in Section IV. However, we resort to simulations in this section since it is not possible to obtain the mean number of successive transmissions from the model due to its memoryless property.

We simulated $N$ nodes sharing a CSMA channel with a propagation delay of $d$ for $N=2,10$ and 100 . For $N=2$, the two nodes are placed with a distance $d$ between them and, for $N=10$ and 100, they are distributed uniformly inside a circle so that the average distance between nodes is $d$. In the back-off mechanism, the probing rate of a node is reduced by $b$ times $(b \geq 1)$ after each transmission. The case $b=1$ corresponds to the pure CSMA case where the transmitter does not reduce its probing rate. We obtained the maximum achievable throughput for each values of $b, b=1,2,4,10$, which maximizes over all possible values of the probing rate. The mean number of successive transmissions are reported at the maximum throughput. Note that the ideally fair mechanism is a TDMAlike channel sharing where the nodes take turns to transmit in which case the mean number of successive transmissions is one. Also note that the successive transmission probability of a node in a fair random access mechanism is $\frac{1}{N}$ which results in $\frac{N}{N-1}$ successive transmissions for a node on the average.

Figs. 11 and 12 plot the maximum throughput and the mean number of successive transmissions for different values of $b$ and $N$ as $d$ increases. The short-term unfairness problem is more apparent for $N=2$ as it becomes less significant for larger $N$ since the mean number of successive transmissions approaches to one. For $N=2$, the fairness improves as $b$ increases. As $b$ increases, the maximum achievable throughput slightly increases for small propagation delays while the throughput slightly reduces for larger propagation delays. For $N=10$ and 100, the short-term unfairness problem is insignificant because some of the randomly placed nodes are close to the transmitting node for large $N$ and these nodes are exposed to the transmission of a node only for a short duration. Yet, the number of successive transmissions reduces as $b$ increases without a degradation in the throughput. For all $N$, 
the fairness degrades as $d$ increases because the duration that other nodes are exposed to a transmission increases and thus the probability that the transmitting node starts a successive transmission increases.

We have evaluated the fairness and throughput performance of the back-off mechanism under saturated traffic conditions and we observe that the back-off mechanism improves the short-term fairness without degrading throughput. The evaluation of the mechanism for a heterogeneous traffic load is a subject of future study. In this case, the performance of the back-off mechanism may not be as desirable as in the case of the saturated traffic. For example, when one of the nodes has traffic and the other nodes are idle, the back-off mechanism will cause an under-utilization of this node by reducing the probing rate of this node after each transmission.

\section{COMPARISON OF THE PROPOSED CSMA MOdEL WITH IEEE 802.11 B CHANNEL ACCESS}

In this section, we evaluate the performance of the CSMA/CA channel access scheme of the IEEE 802.11 protocol in terms of the capacity and the optimum probing rate using simulations. Although the CSMA/CA MAC scheme is different than the pure CSMA scheme modeled in this paper, we wanted to see whether conclusions similar to the ones drawn in earlier sections for the pure CSMA model can be obtained for the 802.11 channel access scheme. We simulated a network scenario where saturated bidirectional User Datagram Protocol (UDP) traffic exists between two nodes that are connected via a $802.11 \mathrm{~b}$ link with a distance $d$. We performed simulations using the ns-2 network simulator [30]. In order to make the comparison more compatible, we disabled the RTS/CTS mechanism of the 802.11 MAC in the simulations. We selected the packet length as 2300 bytes which is close to the maximum frame length in the 802.11 standard. The transmission power of the transmitters are selected sufficiently high so that packets are lost only due to contention. We adjusted the acknowledgement timeout value of the 802.11 standard according to the propagation delay to prevent premature timeouts.

In order to make an appropriate comparison, we calculated the throughput as the ratio of time spent for successful transmissions to total simulation time and we normalized the propagation delay with respect to the packet transmission time. Fig. 13 presents the throughput for the 802.11 protocol along with the optimum throughput obtained from the analytical model proposed for the pure CSMA as the propagation delay increases. Although the throughput of the 802.11 protocol changes in parallel with respect to the optimum throughput obtained for the pure CSMA model, it is below the optimum throughput because of the acknowledgement mechanism. Even when the propagation delay is negligible, the maximum achievable throughput of the CSMA/CA MAC scheme is 0.75 due to the dead period during the transmission of the acknowledgment frame and due to the minimum contention window size which limits the maximum probing rate of the 802.11 MAC.

We also compared the proposed optimum rate analysis against the back-off mechanism of the 802.11 protocol. In addition to the random back-off duration, the inter-transmission

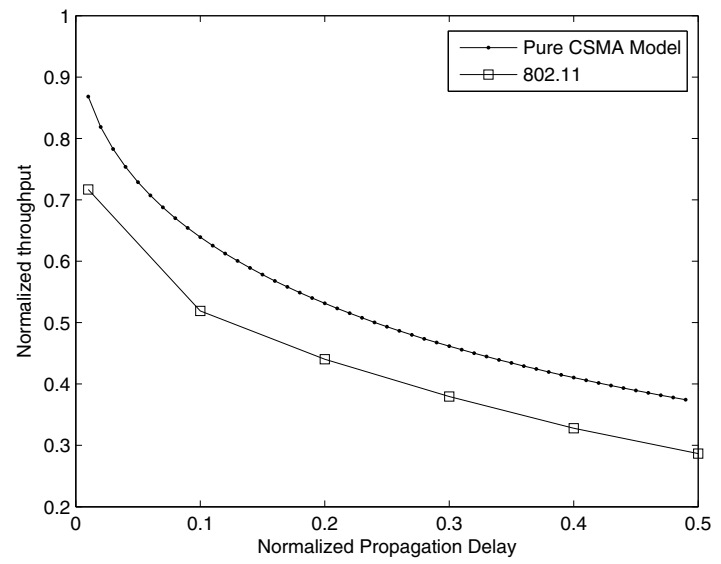

Fig. 13: Throughput of the IEEE 802.11 MAC and the optimum throughput of the pure CSMA model.

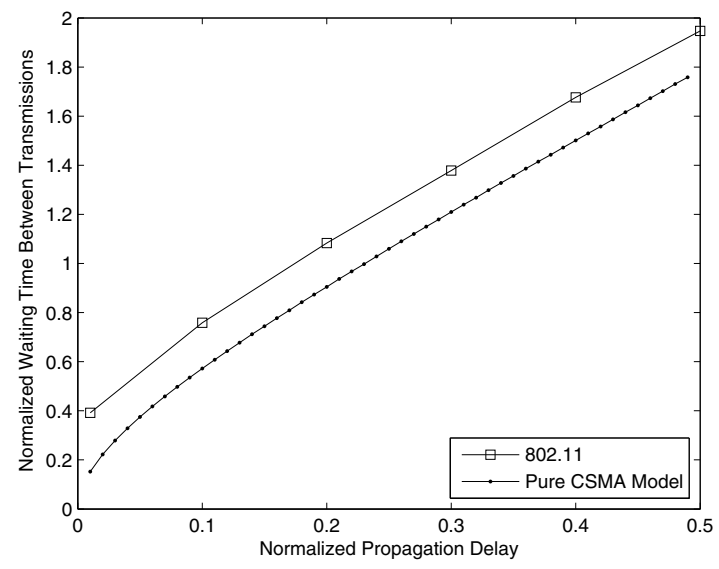

Fig. 14: Mean waiting times between transmissions of the IEEE 802.11 MAC and the pure CSMA model operating at the optimum probing rate.

time between transmissions in the 802.11 protocol includes the waiting time for the acknowledgment and the DCF Interframe Space (DIFS) duration. Because of these fixed durations, the 802.11 random back-off duration is not exactly comparable with the random probing interval of the pure CSMA mechanism considered in this paper. We instead compared the total waiting time between the transmissions in the 802.11 protocol against the total waiting time between transmissions in the pure CSMA mechanism. Fig. 14 presents the normalized waiting time between transmissions for the 802.11 protocol and for the pure CSMA operating at the proposed optimum rate. Waiting time between transmissions are higher in the 802.11 protocol but it behaves in a parallel fashion to the optimum case. The fixed acknowledgment (ACK) timeout duration incorporated in the 802.11 protocol can be accounted for this difference.

It can be concluded that the 802.11 MAC protocol performs in a parallel manner with the proposed model for the pure CSMA in terms of the optimum probing rate and throughput as the propagation delay increases. Although the 802.11 standard adapts the probing rate using the collision information without 
the knowledge of the propagation delay, it performs considerably well for the simulated two-node scenario. In order to improve the performance of the 802.11 protocol under large propagation delays, the acknowledgment mechanism can be eliminated; but a new probing rate adaptation mechanism has to be developed in this case.

\section{CONCLUSIONS}

We modeled the capacity of a single-hop CSMA network when the propagation delays are comparable with the transmission delay. Although large propagation delays are not typical for local area networks, underwater acoustic networks and wireless regional area networks suffer from such propagation delays.

We presented a semi-Markov model for the 2-node case and we derived the capacity and the optimum probing rate expressions for a large number of nodes using this model. We examined how nodes should adapt their aggressiveness in such a CSMA channel. We derived the optimum symmetric rate expression as a function of the average propagation delay, $\bar{d}$, and the number of nodes, $N$. The optimum probing rate for each node decreases asymptotically with $1 / N$ as $N$ increases. On the other hand, the total optimum probing rate achieved by all nodes in the network decreases faster than $\bar{d}^{-1}$ for large $N$.

We have also derived the asymptotic total channel capacity for large networks. According to the proposed model, the total capacity at the optimum rate is asymptotically proportional to $\bar{d}^{-1}$ as the number of nodes, $N$, increases. Despite the increasing number of collisions between nodes, the achieved capacity does not converge to 0 no matter how large the number of nodes in the network is if all nodes in the network probe the channel at the optimum rate.

We have also studied the fairness of the CSMA protocol under large propagation delays and analyzed a back-off mechanism which improves the short-term fairness of the CSMA protocol without a throughput penalty under saturated traffic conditions.

We have compared the proposed capacity and the optimum probing rate analytical model with the performance of the IEEE $802.11 \mathrm{~b}$ channel access scheme using a simple two-node scenario. We observed that the $802.11 \mathrm{~b}$ performs in a similar fashion with the proposed model for the pure CSMA as the propagation delay increases although 802.11 MAC utilizes an acknowledgment mechanism.

\section{REFERENCES}

[1] C. Stevenson, G. Chouinard, Z. Lei, W. Hu, S. Shellhammer, and W. Caldwell, "IEEE 802.22: the first cognitive radio wireless regional area network standard," IEEE Commun. Mag., vol. 47, no. 1, pp. 130138, Jan. 2009.

[2] J. Heidemann, W. Ye, J. Wills, A. Syed, and Y. Li, "Research challenges and applications for underwater sensor networking," in Proc. 2006 IEEE Wireless Communications and Networking Conference, vol. 1, pp. 228235.

[3] Y. Noh, P. Wang, U. Lee, D. Torres, and M. Gerla, "DOTS: a propagation delay-aware opportunistic MAC protocol for underwater sensor networks," in 2010 IEEE International Conference on Network Protocols.

[4] R. Patra, S. Nedevschi, S. Surana, A. Sheth, L. Subramanian, and E. Brewer, "WiLDNet: design and implementation of high performance WiFi based long distance networks," in Proc. 2007 USENIX Conference on Networked Systems Design and Implementation. Available: http://dl.acm.org/citation.cfm?id=1973430.1973437.
[5] E. Lopez-Aguilera, J. Casademont, and J. Cotrina, "Propagation delay influence in IEEE 802.11 outdoor networks," Wireless Networks, vol. 16, no. 4, pp. 1123-1142, May 2010.

[6] L. Jiang and J. Walrand, "A distributed CSMA algorithm for throughput and utility maximization in wireless networks," in Proc. 2008 Allerton Conference on Communication, Control, and Computing, pp. 15111519.

[7] — , "Approaching throughput-optimality in a distributed CSMA algorithm with contention resolution," EECS Department, University of California, Berkeley, Tech. Rep. UCB/EECS-2009-37, Mar. 2009. Available: http://www.eecs.berkeley.edu/Pubs/TechRpts/ 2009/EECS-2009-37.html.

[8] J. Ni, B. Tan, and R. Srikant, "Q-CSMA: queue-length based CSMA/CA algorithms for achieving maximum throughput and low delay in wireless networks," in Proc. 2010 IEEE INFOCOM, pp. 1-5.

[9] J. Liu, Y. Yi, A. Proutiere, M. Chiang, and H. V. Poor, "Towards utilityoptimal random access without message passing," Wireless Commun. and Mobile Computing, vol. 10, no. 1, pp. 115-128, Jan. 2010.

[10] L. Kleinrock and F. Tobagi, "Packet switching in radio channelspart I: carrier sense multiple-access modes and their throughput-delay characteristics," IEEE Trans. Commun., vol. 23, no. 12, pp. 1400-1416, Dec. 1975.

[11] D. Bertsekas and R. Gallager, Data Networks, 2nd ed. Prentice-Hall, Inc., 1992.

[12] H. Takagi and L. Kleinrock, "Throughput analysis for persistent CSMA systems," IEEE Trans. Commun., vol. 33, no. 7, pp. 627-638, Jul. 1985.

[13] K. Leung, B. McNair, L. J. Cimini, and J. Winters, "Outdoor IEEE 802.11 cellular networks: MAC protocol design and performance," in Proc. 2002 IEEE International Conference on Communications, vol. 1, pp. $595-599$.

[14] J. Partan, J. Kurose, and B. N. Levine, "A survey of practical issues in underwater networks," in Proc. 2006 ACM International Workshop on Underwater Networks, pp. 17-24.

[15] H. Matsuno, H. Ishinaka, and T. Shigeyasu, "Effect of propagation delay and RTS packet recognition time on MACA," Electron. and Commun. in Japan (Part I Commun.), vol. 88, no. 1, pp. 21-31, Jan. 2005.

[16] P. Xie and J.-H. Cui, "Exploring random access and handshaking techniques in large-scale underwater wireless acoustic sensor networks," in Proc. 2006 OCEANS, pp. 1-6.

[17] L. Vieira, J. Kong, U. Lee, and M. Gerla, "Analysis of ALOHA protocols for underwater acoustic sensor networks," in 2006 ACM International Workshop on Underwater Networks.

[18] J. Ahn, A. Syed, B. Krishnamachari, and J. Heidemann, "Design and analysis of a propagation delay tolerant ALOHA protocol for underwater networks," Ad Hoc Networks, vol. 9, no. 5, pp. 752766, Jul. 2011. Available: http://www.sciencedirect.com/science/article/ pii/S1570870510001472.

[19] A. A. Syed, W. Ye, J. Heidemann, and B. Krishnamachari, "Understanding spatio-temporal uncertainty in medium access with ALOHA protocols," in Proc. 2007 ACM International Workshop on Underwater Networks, pp. 41-48. Available: http://doi.acm.org/10.1145/1287812. 1287822

[20] S. De, P. Mandal, and S. S. Chakraborty, "On the characterization of Aloha in underwater wireless networks," Mathematical and Computer Modelling, vol. 53, no. 11-12, pp. 2093-2107, Jun. 2011. Available: http://www.sciencedirect.com/science/article/pii/S0895717710003286.

[21] Y. Zhou, K. Chen, J. He, and H. Guan, "Enhanced slotted Aloha protocols for underwater sensor networks with large propagation delay," in Proc. 2011 IEEE Vehicular Technology Conference - Spring, pp. 1-5.

[22] H. Doukkali, S. Houcke, and L. Nuaymi, "A cross layer approach with CSMA/CA based protocol and CDMA transmission for underwater acoustic networks," in 2007 IEEE International Symposium on Personal, Indoor and Mobile Radio Communications.

[23] J. Kurose and K. Ross, Computer Networks: A Top Down Approach Featuring the Internet, 6th ed. Pearson Addison Wesley, 2012.

[24] R. Rom, Local Area and Multiple Access Networks, R. L. Pickholtz, Ed. Computer Science Press, Inc., 1986, ch. Collision detection in radio channels, pp. 235-249.

[25] K. Voulgaris, A. Gkelias, I. Ashraf, M. Dohler, and A. Aghvami, "Throughput analysis of wireless CSMA/CD for a finite user population," in Proc. 2006 IEEE Vehicular Technology Conference, pp. 1-5.

[26] J. Peng, L. Cheng, and B. Sikdar, "A wireless MAC protocol with collision detection," IEEE Trans. Mobile Comput., vol. 6, no. 12, pp. 1357-1369, 2007.

[27] F. Calì, M. Conti, and E. Gregori, "Dynamic tuning of the IEEE 802.11 protocol to achieve a theoretical throughput limit," IEEE/ACM Trans. Networking, vol. 8, no. 6, pp. 785-799, Dec. 2000. 
[28] R. Boorstyn, A. Kershenbaum, B. Maglaris, and V. Sahin, "Throughput analysis in multihop CSMA packet radio networks," IEEE Trans. Commun., vol. 35, no. 3, pp. 267-274, Mar. 1987.

[29] Z. Li, S. Nandi, and A. Gupta, "Modeling the short-term unfairness of IEEE 802.11 in presence of hidden terminals," Performance Evaluation, vol. 63, pp. 441-462, May. 2006.

[30] "Network simulator 2 (ns2)." Available: http://www.isi.edu/nsnam/ns/.

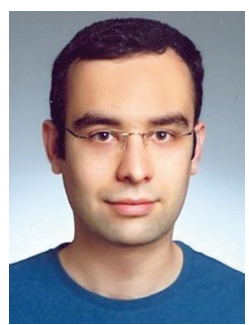

Mehmet Koseoglu received B.Sc. and M.Sc. degrees from the Electrical and Electronics Engineering Department, Bilkent University, Ankara, Turkey in 2004 and 2007, respectively. From to 2004 and 2006, he worked as a software engineer for Aselsan Inc., Ankara, Turkey. He is currently a Ph.D. candidate in Electrical and Electronics Engineering Department of Bilkent University. His research interests are on the performance analysis of wireless multiple access schemes.

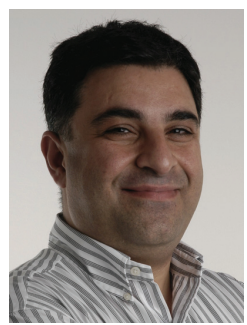

Ezhan Karasan received B.S. degree from Middle East Technical University, Ankara, Turkey, M.S. degree from Bilkent University, Ankara, Turkey, and $\mathrm{Ph} . \mathrm{D}$. degree from Rutgers University, Piscataway, New Jersey, USA, all in electrical engineering, in 1987, 1990, and 1995, respectively. During 19951996, he was a post-doctorate researcher at Bell Labs, Holmdel, New Jersey, USA. From 1996 to 1998, he was a Senior Technical Staff Member in the Lightwave Networks Research Department at AT\&T Labs-Research, Red Bank, New Jersey, USA. He has been with the Department of Electrical and Electronics Engineering at Bilkent University since 1998 , where he is currently a full professor. Dr. Karasan is a member of the Editorial Board of Optical Switching and Networking journal. He is the recipient of 2004 Young Scientist Award from Turkish Scientific and Technical Research Council (TUBITAK), 2005 Young Scientist Award from Mustafa Parlar Foundation and Career Grant from TUBITAK in 2004. Dr. Karasan received a fellowship from NATO Science Scholarship Program for overseas studies in 1991-94. Dr. Karasan has participated in FP6IST Network of Excellence (NoE) e-Photon/ONe+ and FP7-IST NoE BONE projects. His current research interests are in the application of optimization and performance analysis tools for the design, engineering and analysis of optical and wireless networks. 